\title{
Directional synthetic aperture flow imaging using a dual stage beamformer approach
}

\author{
Li, Ye; Jensen, Jørgen Arendt
}

Published in:

Proceedings of 2011 IEEE International Ultrasonics Symposium

Link to article, DOI:

10.1109/ULTSYM.2011.0309

Publication date:

2011

Document Version

Early version, also known as pre-print

Link back to DTU Orbit

Citation (APA):

$\mathrm{Li}$, Y., \& Jensen, J. A. (2011). Directional synthetic aperture flow imaging using a dual stage beamformer approach. In Proceedings of 2011 IEEE International Ultrasonics Symposium (pp. 1254-1257). IEEE. https://doi.org/10.1109/ULTSYM.2011.0309

\section{General rights}

Copyright and moral rights for the publications made accessible in the public portal are retained by the authors and/or other copyright owners and it is a condition of accessing publications that users recognise and abide by the legal requirements associated with these rights.

- Users may download and print one copy of any publication from the public portal for the purpose of private study or research.

- You may not further distribute the material or use it for any profit-making activity or commercial gain

- You may freely distribute the URL identifying the publication in the public portal

If you believe that this document breaches copyright please contact us providing details, and we will remove access to the work immediately and investigate your claim 
Paper presented at the IEEE International Ultrasonics Symposium, Orlando Florida, 2011:

\section{Directional Synthetic Aperture Flow Imaging Using a Dual Stage Beamformer Approach}

Ye Li and Jørgen Arendt Jensen

Center for Fast Ultrasound Imaging,

Biomedical Engineering group, Department of Electrical Engineering, Bldg. 349,

Technical University of Denmark, DK-2800 Kgs. Lyngby, Denmark

To be published in Proceedings of IEEE International Ultrasonics Symposium, Orlando Florida, 2011. 


\title{
Directional Synthetic Aperture Flow Imaging Using a Dual Stage Beamformer Approach
}

\author{
Ye Li and Jørgen Arendt Jensen \\ Center for Fast Ultrasound Imaging, Department of Electrical Engineering, Build. 349 \\ Technical University of Denmark, 2800, Kgs. Lyngby, Denmark
}

\begin{abstract}
A new method for directional synthetic aperture flow imaging using a dual stage beamformer approach is presented. The velocity estimation is angle independent and the amount of calculations is reduced compared to full synthetic aperture, but still maintains all the advantages at the same time. The new method has been studied using the Field II simulations and experimental flow rig measurements. A linear array transducer with $7 \mathrm{MHz}$ center frequency is used, and 64 elements are active to transmit and receive signals. The data is processed in two stages. The first stage has a fixed focus point. In the second stage, focal points are considered as virtual sources and data is beamformed along the flow direction. Then the velocities are estimated by finding the spatial shift between two signals.

In the experimental measurements the angle between the transmit beam and flow vessel was $\mathbf{7 0}^{\circ}$ and a laminar flow with a parabolic profile was generated by a flow rig. The flow with a peak velocity of $0.1 \mathrm{~m} / \mathrm{s}$ was sampled at a pulse repetition frequency of $4 \mathrm{kHz}$. The signals were transmitted and received by the experimental scanner SARUS (Synthetic Aperture Realtime Ultrasound System). A relative standard deviation of $2.3 \%$ and bias of $6.4 \%$ at $65^{\circ}$ were achieved in the simulations, and 4.3\% and $4.2 \%$ for the experimental measurements. A color flow map image was made in $\mathbf{4 8}$ emissions corresponding to a frame rate of 83 frames/s.
\end{abstract}

\section{INTRODUCTION}

The angle dependency of velocity estimates is a major limitation for flow estimation in current ultrasound systems. The data are beamformed along the transmitted ultrasound beam, so only the velocity projected onto the beam direction can be estimated. However, many blood vessels lie along the skin surface that is nearly perpendicular to the transducer surface, which is a major challenge in the clinic.

Many authors have proposed different methods for solving the angle dependency problem. Fox [1] developed a method using multiple crossed-beams to measure the flow velocity and the angle. Trahey and coworkers [2] developed a method for flow imaging based on tracking the motion of the speckle pattern on the B-mode images. Jensen and Munk [3] developed a method that produces a transverse oscillation in the field. Once the lateral modulation is generated in the field, the velocity component in that direction can be estimated. Newhouse and coworkers [4] estimate the multi-dimensional velocity using the spectral broadening phenomenon. The amplitude of echo signals are modulated by the lateral and elevation beam patterns.

The Synthetic Aperture (SA) technique is a solution to this problem. The major advantage of SA is, that the data is available continuously and can be focused dynamically both in transmit and receive [5], [6]. Using recursive imaging, the flow motion can be tracked from the instantaneous high resolution image so the whole color flow map can be created quickly [7]. So the SA technique can be applied to fast flow imaging. Furthermore, the data can be beamformed in any direction with the SA technique, so the vector flow can be obtained by finding the directional signal along the flow direction, and this can be done for any direction. Jensen and Nikolov [8] have investigated this method through simulations, experiments and in vivo measurements. The relative standard deviation can be $0.36 \%$ compared to the peak velocity.

However, full SA requires a large amount of calculations, which gives a high demand on data transfer and storage in the system. Although SA has many advantages against conventional methods, currently it is hard to implement in a scanner. SA dual stage beamforming approach is an alternative method to implement the SA technique. SA using a dual stage beamformer approach is developed for reducing the amount of calculations, but still maintains the advantages of SA [9]. With this method, the angle should be known before the estimation. The angle can be determined based on finding the angle of maximum normalized cross-correlation function [10], so a fully autonomous velocity estimation system can be made. In this paper, results from both simulations and experimental measurements are presented, and a color flow map image is shown. The frame rate is 10 times higher than the conventional method and the amount of calculations is reduced compared to full SA.

\section{Dual Stage Beamformer and Directional BEAMFORMING}

The received signals are processed in two separate beamformers. In the first beamformer, a fixed delay profile is applied on the received data to beamform image lines. In the second stage, the time-of-flight (TOF) is calculated as the distance from the transmit element to the image point via the transmit focus point and back to the receive element via the receive focus point. The data are aligned in phase based on selecting the same TOF and the output data from the first stage is focused dynamically in receive [9]. The beamformed data in the first stage represents the information within the focused ultrasound beam, which is defined by an opening angle $\alpha[9],[11]$

$$
\alpha=2 \arctan \frac{1}{2 F^{\#}}
$$


where $F^{\#}$ is the ratio of the focal point depth to the length of the active sub-aperture.

The directional beamforming is applied in the second stage. Instead of beamforming the data along the ultrasound beam, it is focused along the flow direction. SA can obtain high resolution images (HRIs) continuously, and the data can be focused in any direction. The new coordinate for directional beamforming is shown in Fig. 1

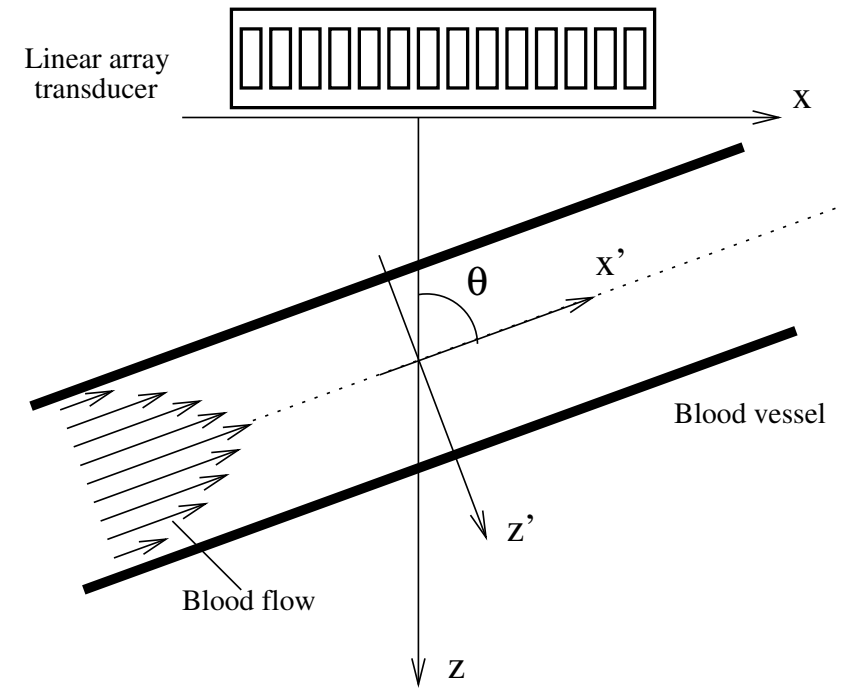

Fig. 1. The setup of the ultrasound imaging system. With directional beamformer, the data is focused along the flow direction instead of the axial direction, so the new coordinate system $\left(x^{\prime}, y^{\prime}, z^{\prime}\right)$ is applied to find positions.

The relations between the new and original coordinates are described as

$$
\begin{aligned}
& x=x^{\prime} \sin (\theta) \\
& y=y^{\prime} \\
& z=z^{\prime} \cos (\theta)-z_{\text {ves }},
\end{aligned}
$$

where $x^{\prime}, y^{\prime}$ and $z^{\prime}$ represent the new coordinates, $z_{\text {ves }}$ is the depth of the vessel, while $x, y$ and $z$ are the original ones. $\theta$ is defined as the angle between the ultrasound beam and the flow direction. Therefore, the focusing points $\vec{r}_{i p}(k)$ can be expressed as

$$
\overrightarrow{r_{i p}}(k)=\left[k \Delta x^{\prime} \sin (\theta), 0, k \Delta z^{\prime} \cos (\theta)+z_{v e s}\right],
$$

where $k$ is the sample index, $\Delta x^{\prime}$ and $\Delta z^{\prime}$ are the spatial sampling interval in the $x^{\prime}, z^{\prime}$ directions.

\section{Flow Velocity ESTIMATOR}

The HRIs are needed to estimate the flow velocity. This can be achieved at every emission, since the data are available continuously and the HRI is formed by summing the last $N$ low resolution images (LRIs). In the new coordinate, the HRI can be formulated as

$$
H^{(n)}\left(\overrightarrow{r_{i p}}(k)\right)=\sum_{i=n-N+1}^{n} L^{(i)}\left(\overrightarrow{r_{i p}}(k)\right),
$$

where $H\left(\overrightarrow{r_{i p}}(k)\right)$ and $L\left(\overrightarrow{r_{i p}}(k)\right)$ are the high resolution images and low resolution images beamformed along the ultrasound beam. $N$ is the number of emission events in one sequence. Two HRIs from the same emission sequence have a spatial shift due to the movement of the scatterer. The relation can be expressed as

$$
H^{(n)}\left(\overrightarrow{r_{i p}}(k)\right)=H^{(n-N)}\left(\overrightarrow{r_{i p}}(k)-\vec{v} T_{p r f} N\right),
$$

where $\vec{v}$ is the velocity of the scatterers and $T_{p r f}$ is the pulse repetition time. The shift term in (5) indicates the speed of the movement over a number of emissions. The cross-correlation technique is used to estimate this shift. As the received signal is sampled at discrete space, the form is

$$
H_{d}(k)=H\left(\overrightarrow{r_{i p}}(k)\right)
$$

Then the cross-correlation function can be calculated in discrete form. Since the cross-correlation function is estimated from two signals at the same depth in the $z^{\prime}$ coordinate, the cross-correlation can be expressed in one dimension. The discrete version is

$$
\begin{array}{r}
R_{n-N, n}(l)=\frac{1}{N_{k}+1} \sum_{k=-N_{K} / 2}^{N_{K} / 2} H_{d}^{(n-N)}(k) H_{d}^{(n)}(k+l) \\
=\frac{1}{N_{k}+1} \sum_{k=-N_{K} / 2}^{N_{K} / 2} H_{d}^{(n-N)}(k) H_{d}^{(n-N)}\left(k+l-k_{s}\right) \\
=R_{n-N, n-N}\left(l-k_{s}\right),
\end{array}
$$

where $N_{k}+1$ is the number of samples in the high resolution signal, $k_{s}$ is the delay lag indicating the peak value in the cross-correlation function. Therefore, the spatial shift can be represented as a delay lag in the cross-correlation function, thus, the speed along the flow direction can be

$$
|\vec{v}|=\frac{k_{s} \Delta z}{N T_{p r f}},
$$

where $k_{s}$ is the delay lag in the cross correlation function, $\Delta z$ is the spatial sampling interval, and $N$ is the number of emissions in one sequence.

\section{RESUlts}

The method was simulated using Field II [12], [13] and verified through experimental measurements. Table I shows the parameters used in both simulations and measurement.

In the simulation, the blood flow is mimicked by numerous moving scatterers with a random uniform distribution and they have random Gaussian distributed amplitudes. 200,000 scatterers are confined in the simulated blood vessel, which is $30 \mathrm{~mm}$ long. Laminar flow with a parabolic profile moves in the vessel, and the peak velocity is $0.1 \mathrm{~m} / \mathrm{s}$.

In the experiment, the flow is generated by a circulating flow rig system as illustrated in Fig. 2. A gear pump drive (ColeParmer, Model: 75211-15) controls the volume flow in the tube. The air-trap device is used to remove air bubbles from the closed circuit and a commercial flow meter Danfoss MAGFLO (Type MAG 3000) is used as the reference for the volume 


\begin{tabular}{lll} 
Parameter & Value & Unit \\
\hline Central frequency & 7 & $\mathrm{MHz}$ \\
Sampling frequency (simulations) & 120 & $\mathrm{MHz}$ \\
Sampling frequency (experiments) & 70 & $\mathrm{MHz}$ \\
Speed of sound & 1480 & $\mathrm{~m} / \mathrm{s}$ \\
Number of elements & 192 & \\
Transducer pitch & 0.208 & $\mathrm{~mm}$ \\
Transducer height & 4.5 & $\mathrm{~mm}$ \\
Pulse repetition frequency & 4 & $\mathrm{kHz}$ \\
Transmit and receive focal depth (virtual source) & 5 & $\mathrm{~mm}$ \\
Number of transmit elements & 64 & \\
Number of receive elements & 64 & \\
The radius of the tube in simulations & 10 & $\mathrm{~mm}$ \\
The radius of the tube in experiments & 6.5 & $\mathrm{~mm}$ \\
\hline
\end{tabular}

TABLE I

PARAMETERS FOR FLOW SIMULATION.

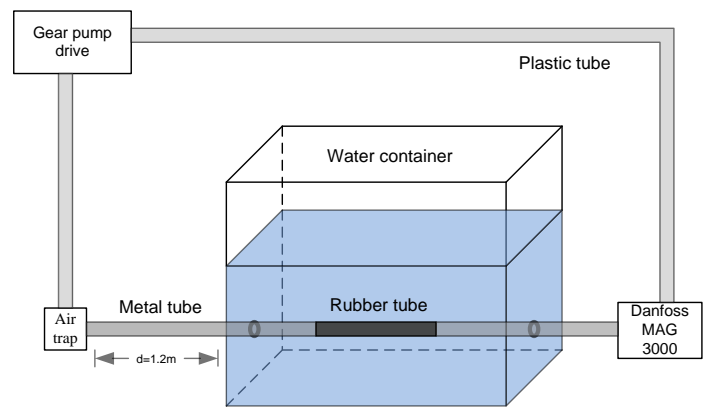

Fig. 2. Flow rig system. The flow is pumped out to the air trap to remove air bubbles, then moves through a $1.2 \mathrm{~m}$ long metal tube to develop a laminar flow. A flow meter measures the volume flow as a reference.

flow. The blood mimicking fluid (Dansk Fantom Service, Frederikssund, Danmark) consists of glycerol, orgasol, Trition x-100 (2\%), NaBenzoat, and $N a_{2} E D T A$ diluted $1: 9$ with demineralized water. Dextran is added to the solution to increase the viscosity, since the flow phantom is intended to mimic blood's properties. The kinematic viscosity of the fluid in the experiment is $3.6 \mathrm{~mm}^{2} / \mathrm{s}$. The flow is maintained with a peak velocity of $0.1 \mathrm{~m} / \mathrm{s}$.

There are four emissions in one sequence, which is repeated several times. The transmission and receive are controlled by SARUS [14] and all the channel data is sampled by SARUS and stored to a disk, and then processed offline.

The simulation results are evaluated through the relative bias and relative standard deviation. The bias is defined as:

$$
v_{\text {bias }}=\frac{1}{v_{\text {peak }} N_{p} N_{v}} \sum_{i=1}^{N_{p}} \sum_{k=1}^{N_{v}} \hat{v}\left(\vec{r}_{k}, i\right)-v\left(\vec{r}_{k}\right),
$$

where $N_{p}$ is the number of estimated profiles, $N_{v}$ is the number of samples in one profile, $\vec{r}_{k}$ represents the position for velocity estimation, $\hat{v}\left(\vec{r}_{k}, i\right)$ is the estimated velocity at $\vec{r}_{k}$ in $i$ th profile, and $v\left(\vec{r}_{k}\right)$ is the true velocity profile. The relative standard deviation is defined as:

$$
v_{\text {std }}=\frac{1}{v_{\text {peak }}} \sqrt{\frac{1}{N_{p} N_{v}} \sum_{i=1}^{N_{p}} \sum_{k=1}^{N_{v}}\left(\hat{v}\left(\vec{r}_{k}, i\right)-\bar{v}\left(\vec{r}_{k}\right)\right)^{2}},
$$

where $\bar{v}\left(\vec{r}_{k}\right)$ is the mean velocity profile estimate. The relative bias and standard deviation are relative to the peak velocity in the vessel.

Fig. 3 illustrates the simulation result, where the angle between the vessel and ultrasound beam is 65 degrees. Each profile is estimated using 48 emissions and there are 13 individual profiles shown in the figure. The relative standard deviation over the profile is $2.3 \%$ compared to the peak velocity and the relative bias is $6.4 \%$. Fig. 4 shows the performance as a function of angles in the simulations. The angle between the ultrasound beam and the vessel varies from $45^{\circ}$ to $85^{\circ}$. A worse performance is obtained with the larger angle because more oscillations in the axial direction than transverse direction can be achieved.

Fig. 5 gives the experimental results from the flow rig measurements. The transmission setup is the same as for the simulations. One sequence consists of four emissions with $7 \lambda$ spacing. 48 emissions are used for one estimation and there are 20 individual profiles in the figure. The relative standard deviation and bias are $4.3 \%$ and $4.2 \%$, respectively.
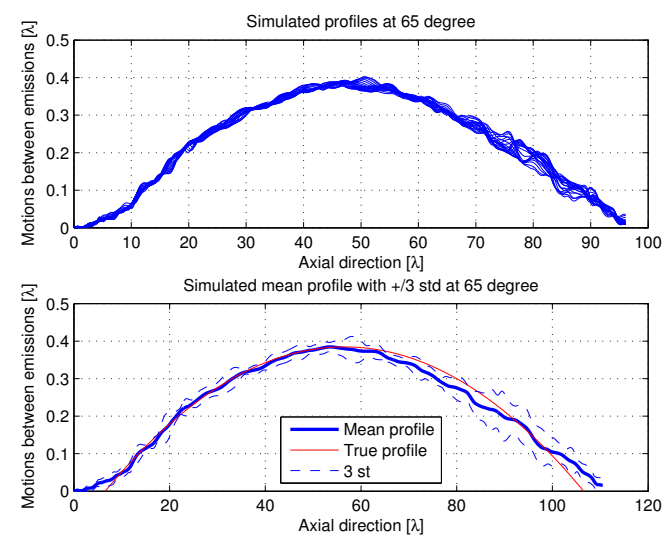

Fig. 3. The angle between flow and ultrasound beam is $65^{\circ}$. The top graph shows 13 individual profiles on top of each other and the lower graph shows the mean profile (solid line) with \pm 3 standard deviation (dashed lines). The red line is the true flow profile for reference.

A color flow image is shown in Fig. 6. The full image is made using 48 emissions and the color scale indicates the flow velocity along the flow direction. The echo canceling is made by finding the mean value of the beamformed signals, and then subtract the mean value from the individual signals. The frame rate obtained in the experiment is 83 frames/s and the measurement depth can be up to $185 \mathrm{~mm}$ according to the $T_{p r f}$ of the system.

\section{Calculations Analysis}

The advantage of this new method is that the amount of calculations are reduced compared to full SA imaging. Full SA needs all the channel data to be used in beamforming one image, which requires a lot of data access and transfer. Current ultrasound systems are not capable of fulfilling these requirements. However, the dual stage beamformer simplify the whole beamforming. The first stage is simple, since a fixed 


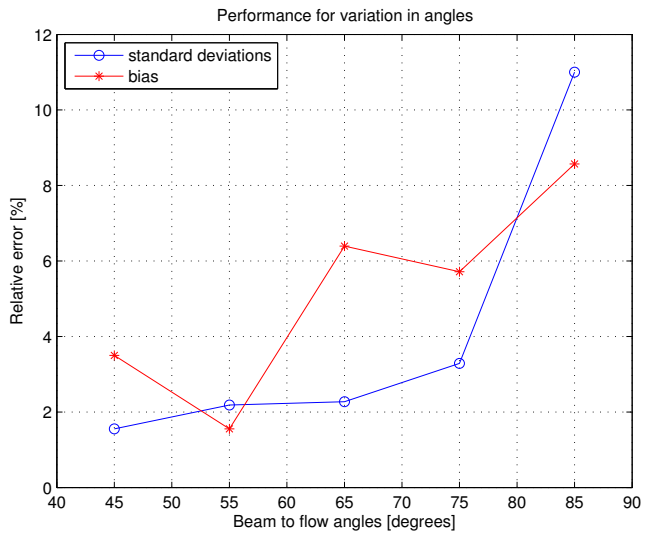

Fig. 4. The performance for various angles. The relative standard deviations and bias are calculated to show the performance of the method.
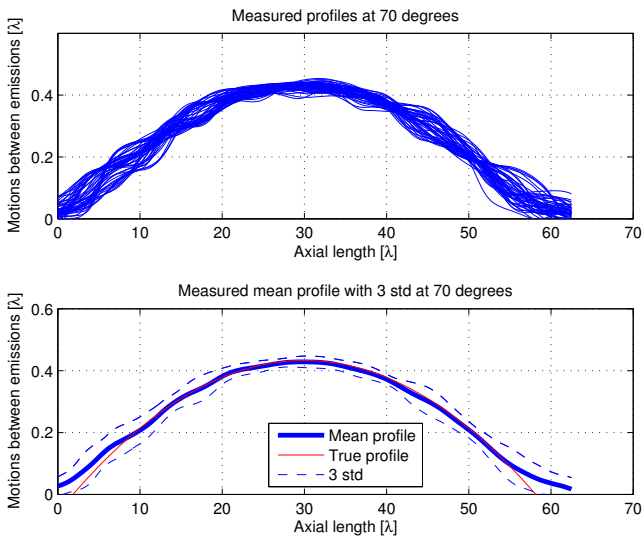

Fig. 5. The estimated velocity profiles at an angle of 70 degrees. Transmit focus point is placed at $5 \mathrm{~mm}$. Top graph shows 20 profiles, and the bottom graph shows the mean velocity profile with \pm 3 standard deviation.

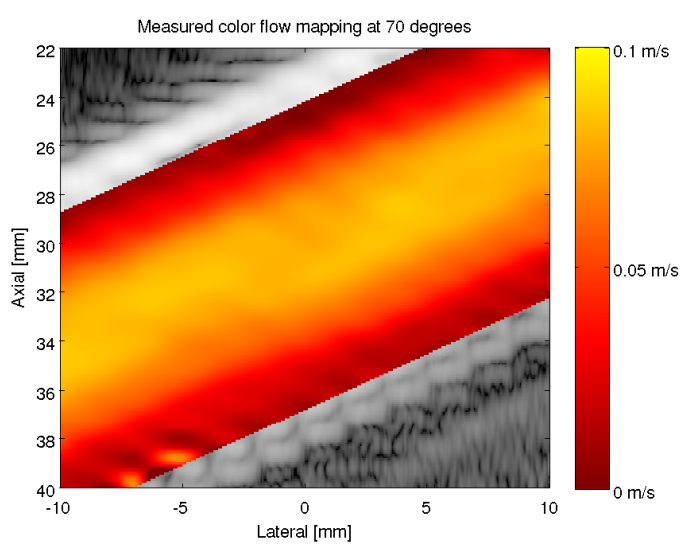

Fig. 6. Color flow map made by the new method. 48 emissions are used for making one image frame. delay profile is used. The second stage requires almost the same amount of calculations as dynamic receive focusing in conventional ultrasound system. This reduces both the memory demands and number of calculations to perform.

\section{DisCUSSION AND CONCLUSION}

The new method for making directional SA flow imaging using a dual stage beamformer approach is presented. The high resolution directional image lines can be achieved continuously due to the continuous available data. The high frame rate of $83 \mathrm{~Hz}$ can be obtained in the presented approach, since HRIs are available at every emissions. The low variance velocity estimates can be achieved, because any number of cross-correlation functions can be averaged to improve the performance. Although the simple mean subtraction echo canceling is applied in this paper, there is no limits to use other more advanced clutter filters, since data is available continuously. The simulations and experiments substantiate that it can be used for directional flow estimation and the amount of calculations for the new method compared to full $\mathrm{SA}$ is reduced.

\section{REFERENCES}

[1] M. D. Fox, "Multiple crossed-beam ultrasound Doppler velocimetry," IEEE Trans. Son. Ultrason., vol. SU-25, pp. 281-286, 1978.

[2] G. E. Trahey, J. W. Allison, and O. T. von Ramm, "Angle independent ultrasonic detection of blood flow," IEEE Trans. Biomed. Eng., vol. BME-34, pp. 965-967, 1987.

[3] J. A. Jensen and P. Munk, "A new method for estimation of velocity vectors," IEEE Trans. Ultrason., Ferroelec., Freq. Contr., vol. 45, pp. 837-851, 1998.

[4] V. L. Newhouse, D. Censor, T. Vontz, J. A. Cisneros, and B. B. Goldberg, "Ultrasound Doppler probing of flows transverse with respect to beam axis," IEEE Trans. Biomed. Eng., vol. BME-34, pp. 779-788, 1987.

[5] L. F. Nock and G. E. Trahey, "Synthetic receive aperture imaging with phase correction for motion and for tissue inhomogenities - part I: basic principles," IEEE Trans. Ultrason., Ferroelec., Freq. Contr., vol. 39, pp. 489-495, 1992.

[6] J. A. Jensen, S. Nikolov, K. L. Gammelmark, and M. H. Pedersen, "Synthetic aperture ultrasound imaging," Ultrasonics, vol. 44, pp. e5e15, 2006

[7] S. I. Nikolov and J. A. Jensen, "In-vivo synthetic aperture flow imaging in medical ultrasound," IEEE Trans. Ultrason., Ferroelec., Freq. Contr., pp. 848-856, 2003.

[8] J. A. Jensen and S. I. Nikolov, "Directional synthetic aperture flow imaging," IEEE Trans. Ultrason., Ferroelec., Freq. Contr., pp. 11071118, 2004.

[9] J. Kortbek and J. A. Jensen, "Synthetic aperture sequential beamforming," in Proc. IEEE Ultrason. Symp., pp. 966-969, 2008.

[10] N. Oddershede and J. A. Jensen, "Synthetic aperture flow angle estimation on in-vivo data from the carotid artery," in Proc. IEEE Ultrason. Symp., pp. 1331-1334, 2005.

[11] N. Oddershede and J. A. Jensen, "Effects influencing focusing in synthetic aperture vector flow imaging," IEEE Trans. Ultrason., Ferroelec., Freq. Contr., vol. 54, pp. 1811-1825, September 2007.

[12] J. A. Jensen, "Field: A program for simulating ultrasound systems," Med. Biol. Eng. Comp., vol. 10th Nordic-Baltic Conference on Biomedical Imaging, Vol. 4, Supplement 1, Part 1, pp. 351-353, 1996 b.

[13] J. A. Jensen and N. B. Svendsen, "Calculation of pressure fields from arbitrarily shaped, apodized, and excited ultrasound transducers," IEEE Trans. Ultrason., Ferroelec., Freq. Contr., vol. 39, pp. 262-267, 1992.

[14] J. A. Jensen, H. H. Lund, R. T. Nielson, B. G. Tomov, M. B. Stuart, S. I. Nikolov, M. Hansen, and U. D. Larsen, "Performance of SARUS: a synthetic aperture real-time ultrasound system," in Proc. IEEE Ultrason. Symp., pp. 305-309, 2010. 